\title{
Korrektur und Nachtrag zu Konvexität der Eigenkurven beim Bénardschen Problem
}

ZAMP 20, Fasc. 5 (1969), Seiten 636-641

Von Waldemar Velte, Institut für angewandte Mathematik, Universität Würzburg, Deutschland

Die auf Seite 638, letzte Formelzeile, und auf Seite 639, erste Formelzeile, angegebenen Ungleichungen für die zweite Ableitung von $\lambda_{1}^{+}$sind nicht richtig. Es müsste zusätzlich der (nicht verschwindende) Term $\partial / \partial \alpha(\delta J / \delta \alpha)$ abgeschätzt werden. Der Satz auf Seite 639, oben, ist zu ersetzen durch folgenden Satz:

Satz. Für alle betrachteten Randbedingungen ist $\alpha \lambda_{1}^{+}(\alpha)$ für $\alpha>0$ eine positive und streng monoton wachsende Funktion von $\alpha$ und auch von $a=\alpha^{2}$. Für $\alpha \rightarrow 0$ und für $\alpha \rightarrow \infty$ ist $\lambda_{1}^{+}(\alpha) \rightarrow \infty$.

Beweis. Mit der Substitution $w=\alpha v, A=\alpha \lambda$ geht (3) über in

$$
L^{2} u=\Lambda w, \quad L w=\Lambda u \text {. }
$$

Das zugehörige Extremalproblem lautet

$$
A_{1}^{+}(\alpha)=\min _{(u, w) \in \mathcal{L}} \bar{J}[u, w, \alpha], \quad \int_{0}^{1} u w d x>0
$$

mit

$$
\vec{J}[u, w, \alpha]=\frac{\int_{0}^{1}\left(u^{\prime \prime 2}+2 \alpha^{2} u^{\prime 2}+\alpha^{4} u^{2}+w^{\prime 2}+\alpha^{2} w^{2}\right) d x}{2 \int_{0}^{1} u w d x} .
$$

Für eine Schar von Eigenfunktionen $\mathrm{zu} \Lambda_{1}^{+}(\alpha)$ folgt dann wegen $\delta \bar{J} / \delta \alpha=0$ für alle $\alpha>0$

$$
\frac{d}{d \alpha} \Lambda_{1}^{+}=\frac{\partial J}{\partial \alpha}=\int_{0}^{1}\left(4 \alpha u^{2}+4 \alpha^{3} u^{2}+2 \alpha w^{2}\right) d x\left(2 \int_{0}^{1} u w d x\right)^{-1}>0,
$$

und analog mit $a=\alpha^{2}$

$$
\frac{d}{d a} A_{1}^{+}=\frac{\partial \bar{J}}{\partial a}=\int_{0}^{1}\left(2 u^{\prime 2}+2 a u^{2}+w^{2}\right) d x\left(2 \int_{0}^{1} u w d x\right)^{-1}>0 .
$$

Daraus folgt die strenge Monotonie von $\Lambda_{1}^{+}=\alpha \lambda_{1}^{+}$.

(Eingegangen: 6. Januar 1970) 